\title{
An Algorithm to Recover Generalized Cylinders from a Single Intensity View
}

\section{CUCS-491-89}

\author{
Ari D. Gross and Terrance E. Boult \\ Deparment of Computer Science \\ Columbia University \\ New York, NY 10027 \\ Ari@cs.columbia.ectu \\ Tboult@cs.columbia.edu
}

October 1989 


\title{
An Algorithm to Recover Generalized Cylinders from a Single Intensity View*
}

\author{
Ari D. Gross and Terrance E. Boult \\ Columbia University Department of Computer Science \\ New York City, New York, 10027 ari@cs.columbia.edu, tboult@cs.columbia.edu
}

\begin{abstract}
Understanding a scene involves the ability to recover the shape of objects in an environment. Generalized cylinders are a flexible, loosely defined class of parametric shapes capable of modeling many real-world objects. Straight homogeneous generalized cylinders are an important subclass of generalized cylinders whose cross sections are scaled versions of a reference curve. In this paper, a general method is presented for recovering straight homogeneous generalized cylinders from monocular intensity images. The algorithm is much more general in scope than any other developed to date, combining constraints derived from both contour and intensity information. We first demonstrate that contour information alone is insufficient to recover a straight homogencous generalized cylinder uniquely. Next, we show that the sign and magnitude of the Gaussian curvature at a point varies among members of a contour-equivalent class. The image contour fails to constrain two parameters required to recover the shape of a generalized cylinder, the 3D axis location and the object tilt. Next, a method for "ruling" straight homogeneous generalized cylinder images is developed. Once the rulings of the image have been recovered, we show that all parameters derivable from contour alone can be recovered. To recover the two remaining parameters (modulo scale) not constrained by image contour requires incorporating additional information into the recovery process. e.g. intensity information. We derive a method for recovering the tilt of the object using the ruled contour image and intensity values along cross-sectional geodesics. In addition, we derive a method for recovering the location of the object's 3D axis from intensity values along meridians of the surface. Using the different methods outlined in this paper constitutes an algorithm for recovering all the shape parameters (modulo scale) of a straight homogeneous generalized cylinder.
\end{abstract}

\section{Introduction}

A generalized cylinder (hereafter GC) is a solid defined by its axis, cross-section, and sweeping rule. Generalized cylinders were first proposed by Binford [1] as a class of parametric shape that is very flexible and capable of modeling many different types of objects. GCs scem general enough to represent many real-world objects yet sufficiently well-defined that we are tempted to recover their shape from image data. They have been the topic of considerable research in computer vision and robotics $([2,3,4,6$, $7,8,9,11,13-17])$.

Exactly because GCs are such an expressive representation, recovery of their shape parameters from image intensity data has proven to be a difficult problem. As a result, focus has shifted towards important subclasses of generalized cylinders. The subclass that has received the most attention is almost certainly that of straight homogencous generalized cylinders, where the axis is straight and cross-section curves are scaled versions of a reference curve (defined in section 2). Even the important subclass straight

\footnotetext{
* Suppon for this work was provided in par by the Advanced Research Projects Agency of the Department of Defense under contract \#. $\$ 00039.84$-C.0165 and by the Vational Science Foundation Grant IRI8800370.
} 
homogeneous generalized cylinders (hereafter SHGCs) has proven difficult to recover from monocular intensity images. Brooks' ACRONYM system [4] was successful at recovering a very restricted subclass of GCs from contour images, and thus cannot be considered a general solution of the generalized cylinder recovery problcm. The subclass considered by Brooks in the ACRONYM system consisted of GCs with a circular or simple polygonal cross section, straight or circular spine, and linear or bilinear sweeping rule. Even with this restricted subset of GCs, ACRONYM was only successful at such recovery of the underlying shapes in the image because it had an a priori set of models it was attempting to match to. This is due to the fact that the contour image alone is insufficient to yield a unique solution, as will be explained later in this paper (section 3).

There have been other attempts at constructing algorithms for the recovery of SHGCs (sce [23],[9],[11-12],[17],[19]). Such attempts either consider a very restricted class of SHGCs (e.g., solids of revolution), rely on heuristic methods, or have an a priori set of models the recovery system is looking for. This is necessarily so, as the general problem of recovering the shape of an SHGC from its contour image is inherently underconstrained and all these methods rely solely on contour information (assuming they use intensity images, as opposed to range images).

In this paper, we provide a method for recovering the unique shape of an SHGC (modulo scale) from its intensity image. This is accomplished by first determining the parameters of shape readily available from SHGC contour. Methods are then developed to recover the remaining parameters, 3D axis location and object tilt, using the SHGC intensity image.

The algorithm presented in this paper relies on some results of other generalized cylinder researchers. In panicular, work done by Steve Shafer [18] and Jcan Ponce [14],[15] have proved extremely helpful to the authors in constructing a complete algorithm for SHGC recovery. In particular, Ponce's algorithm for recovering the SHGC image axis has been incorporated into the larger recovery algorithm.

In adding intensity-based methods (sections 6 and 7) to the recovery algorithm, one has to be concemed that the resulting algorithm will require a detailed a priori knowledge of the imaging model, such as the number of light sources, their positions and intensities, and the lambertain albedo of the surface. Such a restriction is highly undesirable since, except for highly controlled rescarch environments, such information is generally unavailable. We have tried to avoid this by kecping the assumptions as general as possible. For example, the intensity-based method for tilt recovery presented in section 6 makes the following assumptions regarding the imaging model: soft orthographic projection, lambertian reflectance, and constant albedo. The method, however, does not need to know the number of light sources in the imaging model, nor the position and intensity of each light source, nor the lambertain albedo of the matcrial surface. The assumptions are similarly general for the intensity-based method presented in seclion 7 for recovering the 3D axis position.

In this paper, a contour generator is defined as a 3D curve that generates the image contour. There are two kinds of contour generators: limbs, where the surface turns away from the viewer, and edges, where the surface orientation is discontinuous. The 2D contour consists of image limbs and image edges that correspond, respectively, to the 3D limb and edge curves. In this paper, we use the term ruling in a less formal sense than it is used, say, in differential geometry. To rule an SHGC surface (SHGC image) is to draw onto the surface its parallels and meridians (respectively image parailels and image meridians).

This paper avoids some of the assumptions that have been made by generalized cylinder researchers in the past (and have since been shown to be extremely restrictive). In particular, it can be easily shown that the contour generator of an SHGC is not, in general, planar, nor does it lie along a surface meridian, nor is it symmetric with respect to its axis (see [7],[8],[14],[9]). As a result, we make no such assumptions. 
Meridians and parallels, which can be determined directly from the image contour (see section 5), provide a natural parameterization of an SHGC surface and seem to convey considerable information about the underlying shape, e.g. see figure 1. Nevertheless, in sections 3 and 4 of this paper we show that, without additional assumptions, no algorithm can recover the shape of an SHGC from the contour image alone. The underlying ambiguity is shown to have two parameters of freedom, tilt and location of SHGC 3D axis. The ambiguity is significant and can affect the sign and magnitude of Gaussian curvature at a point on the SHGC surface.
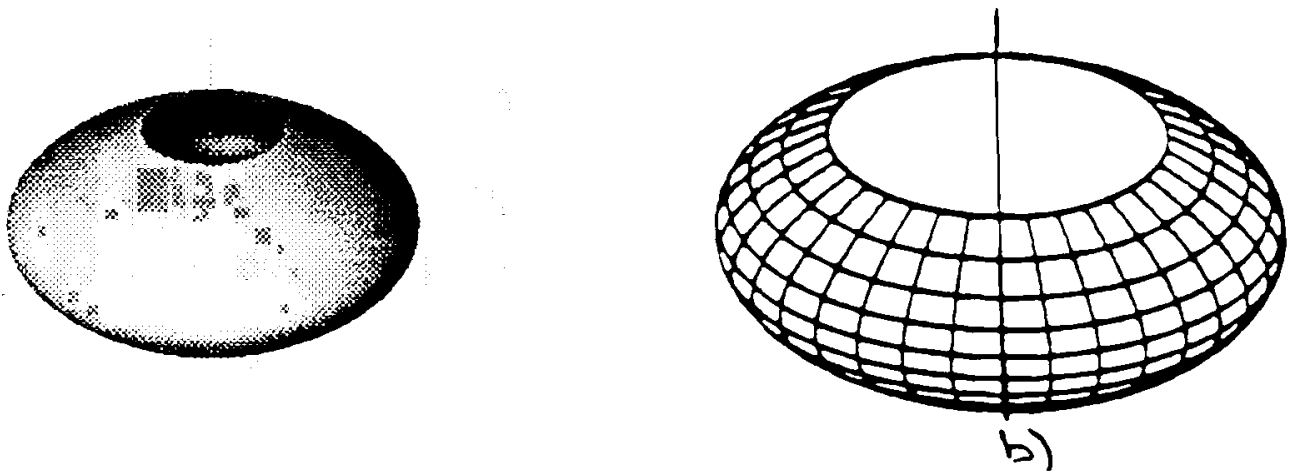

Figure 1. A straight homogencous generalized cylinder: a. the intensity image; b. the ruled surface.

In section 2 of this paper, we define generalized cylinders and straight homogeneous generalized cylinders. Next, we show that there exist classes of SHGCs each member of which can generate the identical contour (section 3). We then show that, among a given class of contour-equivalent SHGCs, the Gaussian curvature at a given point on the SHGC surface can vary both quantitatively and qualitatively, e.g., the surface region can be elliptic or hyperbolic (section 4). In section 5, we develop a method for ruling the image of an SHGC and show that from the image of a ruled SHGC all parameters of the underlying surface constrained by the contour image can be computed (e.g., the sweeping rule at an image point). In section 6, a method is given for disambiguating among members of a contour-equivalent class of SHGCs. This method uses the intensity information along an SHGC cross-sectional geodesic to recover the tilt angle of the object with respect to the viewer reference frame. Finally, a method is presented for recovering the 3D axis position using intensity values at 2 pairs of surface meridian points (section 7). Combining the methods described in sections 5, 6, and 7 provides a complete algorithm for recovering the unique shape of an SHGC (modulo scale) from a monocular intensity image.

\section{Generalized Cylinders: Definitions and Assumptions}

First, we present a definition of generalized cylinders as it will be used in this paper.

Definition: A generalized cylinder is the solid swept by a planar cross-section as it is moved and deformed along an axis.

This definition is very general and includes some ill-defined shapes (c.g., self-intersecting). Consequently, no system has yet been developed for the recovery of shape parameters for general GCs without additional assumptions. For a taxonomy of Generalized Cylinder subclasses see [18].

\subsection{Straight Homogeneous Generalized Cylinders}

In this paper, we are interested in the subclass of generalized cylinders known as straight homogeneous generalized cylinders. SHGCs have been defined with varying degrees of generality in the literature. Typically, the more general a definition adopted for the SHGC class, the less that can be said about its projective invariant properties and consequently, the harder it is to develop a recovery algorithm. We 
first present intuitive definitions of some subclasses, followed by the corresponding algebraic formulae.

Definition: An SHGC is a GC with the following properties: the cross-sections are orthogonal to their axis; the cross-sections are deformed only by scaling, the amount of scaling can be parameterized as a function of the distance along the axis; and the cross-section curve is assumed to be twice continuously differentiable.

We define the GC's with respect to the orthonormal coordinate system $(O, \vec{i}, \vec{j}, \vec{k})$, where $O$ is a point on the axis, and $(\vec{i}, \vec{j})$ is a vector basis of the reference cross-section plane. We define SHGCs by the equation:

$$
O P(z, t)=f(z) p(t) \vec{i}+f(z) q(t) \vec{j}+z \vec{k}
$$

where the function $f$ is the sweeping rule of the SHGC and $p$ and $q$ are the parameterization of the cross-section curve in the $\vec{i}$ and $\vec{j}$ directions, respectively. Note that this definition does not require the SHGC axis to be contained within the closed cross-section curve. SHGCs with the axis extemal to the cross-section curve do not always "appear to" have a straight axis, c.g., see figure 2.

Since everything is up to a scale factor, we set the scale by defining the scaling function of the top cross section curve to $f_{0}=1$. Curves on the SHGC surface of constant $t$ are called meridians while curves of constant $\mathrm{z}$ drawn on the SHGC surface are called parallels. This terminology is analogous to that used for solids of revolutions. We assume that both the sweeping rule function and the cross-section curve are twice continuously differentiable $\left(C^{2}\right)$.

In this paper, we will show that a class of SHGCs may produce the identical contour while varying in Gaussian curvature both quantitatively and qualitatively. Some examples of this are given in figure 2. In figure $2 \mathrm{a}, 2 \mathrm{~b}$, and $2 \mathrm{c}$ we show three examples of SHGCs that are all capable, if seen from the proper viewing position, of producing the contour shown in figure 1 . It is clear that these three shapes differ in their qualitative Gaussian curvature for cerain regions of their respective surfaces. The same section that is elliptic for the shape shown in figure 2 a can clearly be seen to be respectively parabolic and hyperbolic in figures $2 b$ and $2 c$.
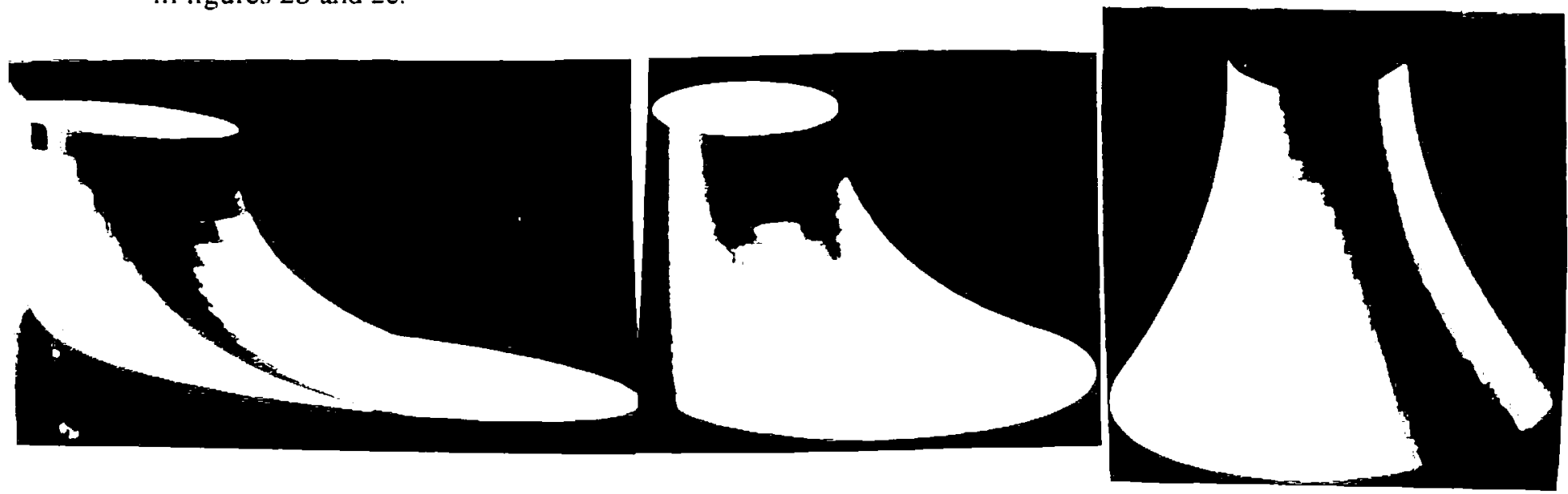

Figure 2. Example of 3 contour-equivalent SHGCs with different Gaussian curvature: a. elliptic region; b. parabolic region; c. hyperbolic region.

\subsection{The Coordinate System}

Consider an SHGC originally aligned with the viewer reference frame, where the viewer reference frame is given by the onhonormal basis $(\vec{u}, \vec{v}, \vec{w})$. The SHGC is parameterized in its own coordinate syslem, having an object-centered onthonormal basis $(\vec{i}, \vec{j}, \vec{k})$. The SHGC is originally in canonical position 
vis a vis the viewer reference frame, i.c., the vectors $\vec{u}$ and $\vec{k}, \vec{v}$ and $\vec{i}$, and $\vec{w}$ and $\vec{j}$ are respectively parallel (see figure 3.a). Assume the SHGC $\overrightarrow{O P}$ is then rotated in space; the rotated SHGC can be parameterized by

$$
O \vec{P}^{\prime}=R_{\diamond} R_{\psi} R_{\omega} O \vec{P}
$$

where $\phi, \psi$, and $\omega$ are the Euler angles expressing the rotation about the $v, w$, and $u$ axes respectively, and $R_{0}, R_{\psi}$ and $R_{\omega}$ are the rotation matrices about the $v, w, u$ axes respectively. Clearly, the initial rotation around the $u$ axis can be ignored as the resulting SHGC can also be considered in canonical alignment with the $(\vec{a}, \vec{v}, \vec{w})$ viewer-centered reference frame, where the initial cross section functions $p$ and $q$ are replaced with the new cross-section functions $p^{\prime}$ and $q^{\prime}$. The last rotation, around the $v$ axis, rotates the projected contour in the image plane but does not modify it in any other way. This image plane rotation can be reversed by finding the image axis, which is a projection of the object-centercd $\vec{k}$ axis onto the image plane, and undoing the $R_{\diamond}$ rotation by bringing the image axis into alignment with (parallel to) the viewer-centered $\vec{u}$ axis. Thus, without loss of generality, we will only study an SHGC rotated towards or away from the viewer, i.e.. around the $\vec{w}$ axis (sce figure 3.b). We refer to this rotation around the $\vec{w}$ axis as the tilt of the SHGC.
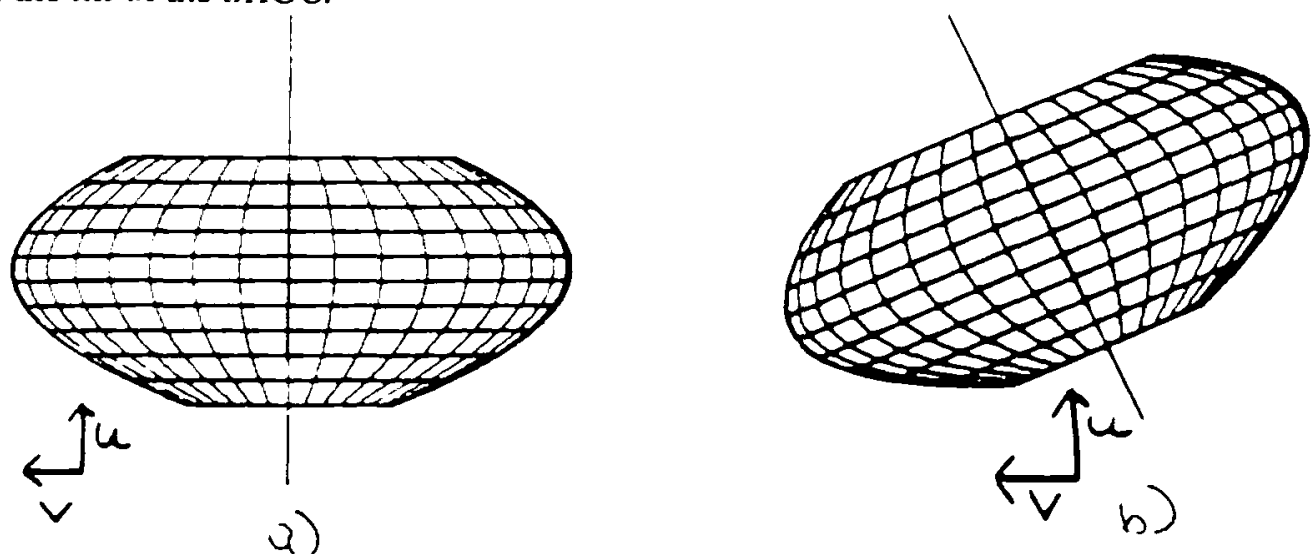

Figure 3. The coordinate system used to define a straight homogencous generalized cylinder: a. in canonical position; $b$. tilted in the viewer direction.

Suppose the viewing direction $\vec{v}$ is given by its spherical coordinates $(\alpha, \beta)$ in $(O, \vec{i}, \vec{j}, \vec{k})$. Based on the proceeding argument, without loss of generality $\alpha$ can be set equal to zero. The resulting orthonormal basis of the viewer reference frame $(\vec{u}, \vec{v}, \vec{w})$ is defined by

$$
\vec{u}=-\cos \beta \vec{i}+\sin \beta \vec{k}, \quad \vec{v}=\sin \beta \vec{i}+\cos \beta \vec{k}, \quad \vec{w}=\vec{j}
$$

Consider the image of an SHGC for some viewing angle, $0<\beta<\pi / 2$, as shown in figure 3.b. It can be easily seen that each projected cross-section curve is a scaled version of the projected reference curve. This will prove useful for ruling the image surface (section 5).

\section{Contour-equivalent classes}

Considerable research has been devoted to the problem of recovering the shape of an SHGC from its contour. However, there has been less focus on the problem of determining exaculy how the contour constrains the class of SHGCs that could have produced it. Looking at both the intensity and contour SHGC images displayed in figure 1, one gets a strong fecling for the underlying shape. What constraints are imposed on the SHGC shape by the contour image and what constraints are imposed on the SHGC by the intensity image? With respect to constraints on the SHGC shape imposed by contour, it can be shown [7] 
that contour-equivalent classes exist among SHGCs, where each member of the class is capable of generating the same contour. This was illustrated in the previous section (e.g., figures $2 \mathrm{a}, 2 \mathrm{~b}, 2 \mathrm{c}$ ).

One can parameterize the contour equivalence classes with two independent parameters. These parameters correspond to the tilt angle with respect to the viewer reference frame, and the image point corresponding to the point of intersection between the SHGC axis and the cross-section plane. In the next two sections, we formally define these two contour-equivalent SHGC classes. After these two classes are defined, the shape propertics of members in each class are considered. In particular, the Gaussian curvature of a surface point (corresponding to a given point on the image) is computed to determine both its qualitative and quantitative variation.

\subsection{Tilt contour-equivalent SHGCs}

The definition we use for SHGCs is again given by

$$
\overrightarrow{O P}(z, t)=f(z) p(t) \vec{i}+f(z) q(t) \vec{j}+z \vec{k}
$$

Now consider a particular SHGC $S_{0}$, defined as in equation (3.1). We are concemed with the contour (both image limbs and edges) produced by SHGC $S_{0}$, having sweeping rule $f_{0}(z)$ and cross-sectional $\vec{i}$ and $\vec{j}$ components of $p_{0}(t)$ and $q_{0}(t)$ respectivcly, with tilt angle $=\beta$. We will refer to this contour as $C_{S_{\text {, }} \beta}$.

We are now interested in defining a class of SHGCs, each member of which is capable of producing a contour identical to the one generated by $S_{0}$ when viewed with tilt angle $=\beta$. The intuitive idea here is to take the original cross-section curve for $S_{0}$, where it is aligned canonically with the vicwer's line of sight (as in figure 3.a), and stretch it along the viewing direction $\vec{v}$ (which initially is parallel to $\vec{i}$ ). We then tilt it towards (or away) from the viewer until a contour identical to $C_{S_{0}, \beta}$ is generated. This motivates our definition for the family of SHGCs that are tilt contour-equivalent to $S_{0}$, where $S_{i}$ is tilt contourequivalent to $S_{0}$ iff $C_{S_{2}, \beta}=C_{S_{1}, \gamma_{i}}$. We define $S_{i}$ as follows:

$$
\overrightarrow{S_{i}}(s(z), t)=r(s) k p_{0}(l) \vec{i}+r(s) q_{0}(t) \vec{j}+s(z) \vec{k}
$$

where $k=\frac{\cos \beta}{\cos \gamma_{i}}, s(z)=z \frac{\sin \beta}{\sin \gamma_{i}}, k \geq 1$, and $r(s(z))=f(z)$. k can be viewed as the stretching factor, i.e., the factor by which the cross-section curve for $S_{i}$ has been stretched in the $\vec{i}$ direction.

It can be proven [7] that, for any stretching factor $\mathrm{k} \geq 1$, the viewing angle $\gamma_{i}$ and the SHGC $S_{i}$, defined in equation (3.2), produce an image contour $C_{S_{,}, \gamma}$ such that $C_{S_{1}, \gamma}=C_{S_{,}, \beta}$, i.e., the SHGCs $S_{0}$ and $S_{i}$ are tilt contour-equivalent. That being truc, we cannot ascertain more about the underlying SHGC, given a monocular image contour (without heuristics), than that it is a member of the contour-equivalent class defined above.

\subsection{Axis-translated contour-equivalent SHGCs}

We now define a second family of SHGCs, generated by varying the second parameter of freedom (unconstrained by the image contour). This second parameter is the location of the SHGC axis, i.e., where the axis intersects the cross-section plane. We assume the image axis can be uniquely determined from the contour. However, this still leaves latitude for translating the SHGC axis in the object-centered $\vec{i}$ direction, i.e. to change the parameter $t_{x}$.

Let us again consider a paricular SHGC $S_{0}$, defined as above. Again, we are concerned with the contour (both limbs and edges) produced by SHGC $S_{0}$, having sweeping rule $f_{0}(z)$ and cross-sectional $\vec{i}$ and $\vec{j}$ components of $p_{0}(t)$ and $q_{0}(t)$ respectively, with tilt $=\beta$ and translation of the reference crosssection curve with respect to the axis $=0$. We refer to this contour as $C_{S_{,}, \beta, 0}{ }^{1}$

1. The 3rd parameter of the subscript denotes the amount the reference cross-section curve has been translated along the cross-section plane in the positive $\vec{i}$ direction. 
We are now interested in defining a class of SHGCs, each member of which is capable of producing a contour identical to the one produced by $S_{0}$ when viewed from the same viewing angle, i.c., $\beta$, but where the location of the axis is allowed to vary. The intuitive idea here is to take each cross-section curve of the original SHGC $\left(S_{0}\right)$, translate it with respect to the axis, and then slide it up or down the axis to achieve a contour equivalent to that of $C_{S_{\mathrm{s}} \beta, 0}$. This then motivates our definition for the family of SHGCs that are axis-translated contour-equivalent to $S_{0}$, where $S_{i}$ is considered axis-translated contourequivalent to $S_{0}$ iff $C_{S_{0}, \beta, 0}=C_{S_{1}, \beta, h}$. We define $S_{i}$, a member of this contour-equivalent class, as

$$
\overrightarrow{S_{i}}(s(z), t)=r(s)(p(t)+h) \vec{i}+r(s) q(t) \vec{j}+s(z) \vec{k}
$$

where $h$ is the distance the reference cross-section curve has been translated from the origin $O$ in the $\vec{i}$ direction, and $s(z)=z-\frac{h\left(1-f_{z}\right)}{\tan \beta}$, where $f_{z}$ is the sweeping rule of SHGC $s_{0}$.

It can be proven [7] that, for any axis translation factor $h$, the sweeping rule $r(s)=f(z)$, where $s$ is a

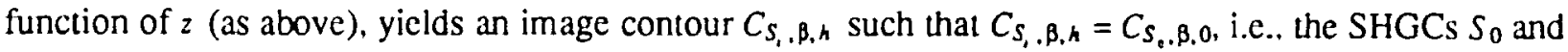
$S_{i}$ are axis-translated contour-equivalent.

Thus, without heuristic methods, we are confronted with the fact that the contour of an SHGC image still leaves us with classes of contour-cquivalent SHGCs. In particular, one class of contourequivalent SHGC shapes allows for changing of the viewing direction and stretching the cross-section function. The other contour-equivalent class fixes the viewing angle but varies the location of the crosssection curves with respect to the axis (as well as the corresponding sweeping rule).

\section{Gaussian curvature for equivalent contour classes}

We now consider the Gaussian curvature of a point on an SHGC surface that maps into a given point in the image. Since we have defined classes of SHGCs that map into the same contour, one can ask how the Gaussian curvature changes at a surface point as the shape of the SHGC is allowed to change (restricted to members of a contour-equivalent class). A study of how the curvature of the contour affects the Gaussian curvature of points along the contour generator can be found in [10]. Here we are concemed with how the Gaussian curvature of surface regions visible in the image are constrained by contour.

\subsection{Tilt equivalent contour-equivalent class: Gaussian curvature}

Where SHGC $S$, is defined as in equation (3.2) (i.e., the SHGC is a member of the tilt contourequivalent class), the Gaussian curvature can be shown (see [5] and [7]) to be given by

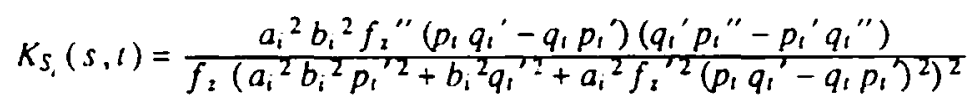

where $a_{i}=k_{i}=\frac{\cos \beta}{\cos \gamma_{i}}$ and $b_{i}=\frac{\sin \beta}{\sin \gamma_{i}}$.

It can been seen from this equation that as stretching factor $k_{i}$ changes so does the Gaussian curvaturc. In fact, the Gaussian curvature at a point on this surface goes to zero as $k \rightarrow \infty$. The Gaussian curvature will tend towards $\infty$ at a point where $f_{z}{ }^{\prime}=0, p_{1}{ }^{\prime}=0$, and $k \rightarrow \infty$. It can also be determined from equation (4.1) that the Gaussian curvature of the surface point can be made to vary quantitatively but not qualitatively; i.e., the sign of the Gaussian curvature will nol vary at a given surface point for any member of the tilt contour-equivalent class of SHGCs. 


\subsection{Axis-translated contour-equivalent class: Gaussian curvature}

Next, we consider the Gaussian curvature of a point on an SHGC surface that is a member of the axis-translated contour-equivalent SHGC class and that maps into a given point in the image. Where SHGC $S_{i}$ is defined as in equation (3.3), the Gaussian curvature can be shown (see [5] and [7]) to be given by

$$
K_{S}(s, l)=\frac{d_{i}^{3} f_{z}^{\prime \prime}\left(\left(p_{t}+h_{i}\right) q_{t}^{\prime}-q_{t} p_{t}\right)\left(q_{t}^{\prime} p_{t}^{\prime \prime}-p_{t}^{\prime} q_{t}^{\prime \prime}\right)}{f_{2}\left(p_{t}^{\prime 2}+q_{t}^{\prime 2}+d_{i}^{2} f_{z}^{\prime 2}\left(\left(p_{t}+h_{i}\right) q_{t}^{\prime}-q_{t} p_{t}^{\prime}\right)^{2}\right)^{2}}
$$

where $d_{i}=\frac{\tan \beta}{\tan \beta+h_{i} f_{2}}$ and $h_{i}$ is the translation of the reference cross-section curve from the axis for SHGC $S_{i}$.

It can be readily seen from this equation that as the translation factor $h_{i}$ changes so does the Gaussian curvature. In fact, the Gaussian curvature at a point on the surface goes to zero as $d_{i} \rightarrow \infty$; this occurs when $\tan \beta=-h_{i} f_{z}$. Also, it can be scen that $K \rightarrow 0$ as $h \rightarrow \infty$. Because of the $d_{i}{ }^{3}$ term in the numerator of the equation, it is clear that by varying $h$ the Gaussian curvature can be made to change not only quantitatively but also qualitatively (positive to negative or the reverse).

The quantitative change in Gaussian curvature as the SHGC surface changes is not necessarily a good indicator of how much one SHGC shape differs from another. This is due, among other things, to a lack of scale invariance in the measure (see [20]). To get additional information on SHGC surface variation, the range of possible normal directions at a point on the surface can be computed (see [7]).

\section{Ruling over generalized cylinders}

There are a finite number of ways that an image cross-section can interact with the image contour. By studying the possible interactions, we derive a method for ruling the surface of an SHGC. i.e., for being able to detect the image meridians and parallels from the contour image. We refer to the detection of image parallels and meridians on the image of an SHGC surface as a ruling over the image of the surface, as described in section 1.

In general, one does not see an object from a degenerate view, i.e., directly from above or directly from the side. Assuming this to be true. the SHGC cross-section curve does not project onto a straight line in the image plane, but rather onto a closed planar curve (which may include straight line segments). We are interested in ruling the SHGC image assuming the image contains both image limbs and image edges.

There are different ways in which an image cross-section can affect the SHGC bounding contour. It is possible for image cross-sections not to intersect the bounding contour at all. It is also possible for an image cross-section to intersect this contour at exactly one point. Finally, an image cross-section can intersect the bounding contour at two or more points. Examples of each configuration are given in [7].

In general, most non-occluded image cross-sections tend to have 2 or more points of intersection with the contour, which suggests a method for ruling the SHGC image. This method assumes the image axis has been recovered. Algorithms using the SHGC contour to recover the image axis of an SHGC, where the cross-section function is assumed polar with respect to the axis, are given in [14] (though the robustness of such algorithms is not assured). In [7], we prove that the 2D axis lemma, on which the algorithm for recovering the image axis is based. generalizes to SHGCs with arbitrary cross-section functions, as defined in Equation (3.1). Once the image axis has been recovered, the reference curve is placed at a given point along the image axis and scaled so that it touches the bounding contour at 2 or more points, without any point extending beyond this contour. Using a non-accidental alignment criterion, we assume that if the above scaling exists, it indicates that the image parallel at this point (along the axis) has been correctly recovered. The alternative, that the image cross-section at this point does not actually 
contribute to the contour and it is only coincidental that the scaled image cross-section intersects the contour at two or more points (without extending beyond it), is ruled out as an unlikely occurrence. This allows us to draw image parallels at any desired point along the image axis.

This technique is illustrated in figure 4 which shows the contour and image axis of the same SHGC shown in figure 1. Also shown in the figure are scaled versions of the reference cross-section curve for a certain point along the image axis. It can be seen that for only one scaling the image cross-section curve exactly touches the contour in two places; for every other scaling, the cross-section curve is either contained entirely within or extends beyond the bounding contour. Thus, this osculating image parallel is presumed to be the correct scaling of the projected reference cross-section curve at this point along the image axis. ${ }^{2}$

Once the image parallels have been found at various locations along the image axis, the image meridians can be computed by connecting corresponding points of adjacent parallels with smooth interpolating curves. The possible parameterizations of an SHGC can be grouped into equivalence classes, as explained in [15]. That being the case, we can decide on a particular parameterization from among this equivalence class by, somewhat arbitrarily, setting the scaling function of the top cross section curve to $f_{0}=1$. Having done so, it is clear from equation (2.4) that the scaling factor is also known for all the image parallels detected by the method described above.

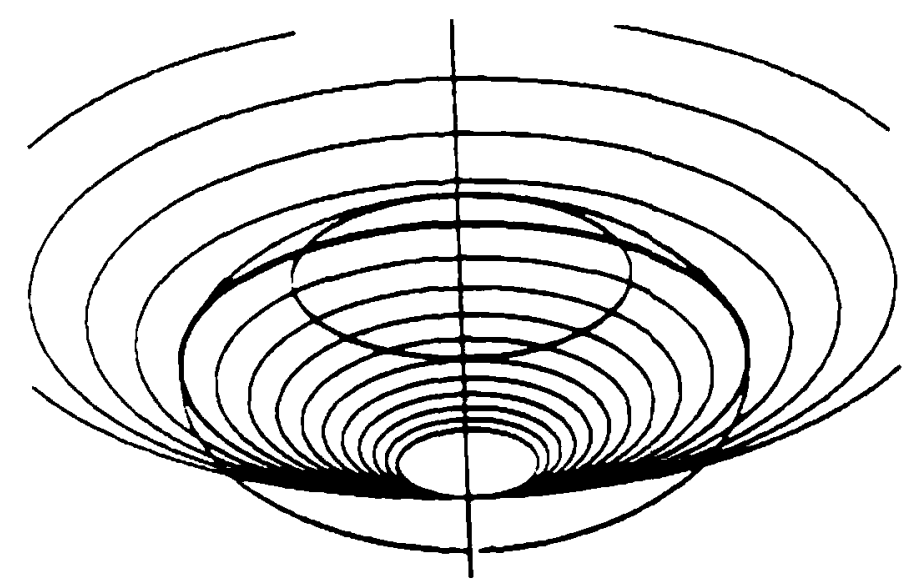

Figure 4. A method for ruling the SHGC image: different scalings of the image parallel and the correct scaling.

\section{Solving for the tilt angle}

In the preceeding section, we derived a method for ruling the SHGC contour. The parameters that remain unconstrained by ruling the SHGC image are the tilt angle and the 3D axis location. This is clear since the ruling of the SHGC contour is completely a function of the SHGC contour, and the SHGC contour alone is insufficient to constrain these two parameters, as explained in section 3 . We then showed, in section 4 , how varying these free parameters can affect the qualitative and quantitative Gaussian curvature of the SHGC surface.

2. This method fails to recover the scaled image parallel at a point along the image axis where the image parallel intersects the contour at al most one point. In addition we note that it cannot be determined from the contour to which point on the image axis this image cross-section curve corresponds. That correspondence depends on where the image axis intersects the image parallel which cannot be ascertained from contour information alone, as demonstrated in section 3 of this paper. 
The logical question that follows from the previous sections is: "given that these contour-equivalent classes exist, how does one recover a unique SHGC from an SHGC image?". The answer is to either rely on contour information alone and incorporate heuristics into the recovery process (extremum principle. etc.), or to incorporate additional information into the recovery process. The most immediate source of additional information (and consequently of additional constraints on the solution space) is from the SHGC intensity image. Such additional constraints can be used to solve for the tilt angle (towards viewer) and the $3 \mathrm{D}$ axis position.

A method of solving for both of these parameters, tilt and axis translation, is presented in [7] . ${ }^{3}$ We describe both of these methods briefly in this section and the next. Once these parameters are recovered, the surface can be completely described (modulo scale). ${ }^{4}$

We now describe, in this section, the method for recovering the object tilt by incorporating intensity information into the recovery process. Assume we have an SHGC intensity image, as shown in figure 5.a. First, the image axis is recovered (see [14]). Next, we find contour points that are extrema of distance between the SHGC contour and the image of its axis. Due to a lemma by Ponce (see [14]), we know that this contour distance extrema corresponds to an extrema of the sweeping rule function $f$. But this means that such points are projections of geodesic curves, where the tangent vector along the meridian is parallel to the SHCG axis.

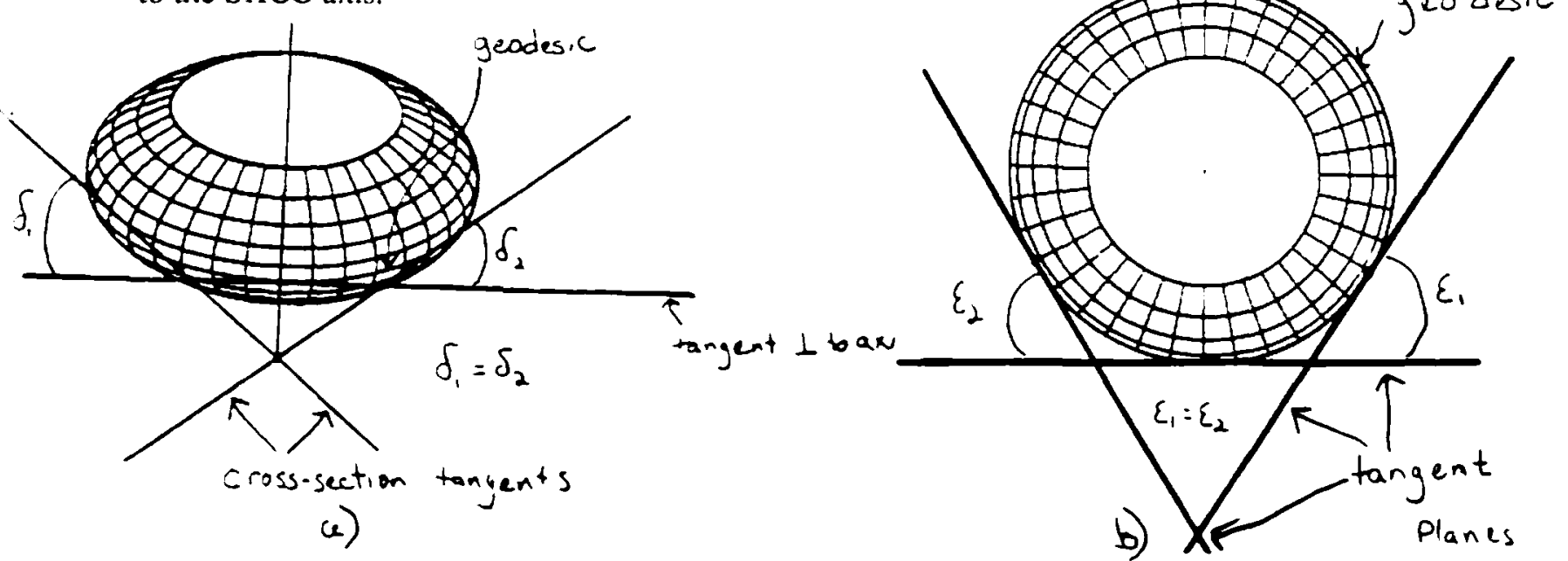

Figure 5. SHGC tilt recovery method: a. drawing of image cross-section tangents; b. overhead view of tangent planes intersecting for points on the cross-section curve.

Next, using the method described in section 5, we recover the image parallel at one of the contour extrema points (lying on a geodesic of the SHGC). As a corollary of the mean value theorem, there exists at least one point on this image parallel with an image tangent vector along the image parallel curve that is perpendicular to the image axis. We will refer to this image point (as seen by the camera) as $C_{0}$. Next, we find a point on each side of $C_{0}$. such that the tangents to the image parallel at these points intersect the tangent $t_{0}$ at $C_{0}$ at opposite angles, $\theta$ and $\pi-\theta$ respectively. We will refer to these points as $C_{r}$ and $C_{l}$; see figure 5.b. Let $S_{0}, S_{1}$, and $S_{l}$ be points on the surface of the SHGC that correspond, respectively, to the image points $C_{0}, C_{r}$, and $C_{l}$. It can be shown [7] that the tangent planes at $S_{r}$ and $S_{l}$ make equal angles with the tangent plane at $S_{0}$ (modulo the sign of the angle); see figure 5.b.

3. The method requires making some general assumptions regarding the imaging model, e.g., orthographic projection, lambertian reflectance, and constant lambertain albedo.

4. This recovery method also requires that the SHCG have an extrema of the sweeping rule (c.g.. geodesic cross-section curve). 
Consider a single light source at some arbitrary position in space, though distant from the SHGC object (i.e., projection is onthographic). We now select a new object-centered coordinate system for the SHGC, obtained by rotating the $\vec{i}, \vec{j}, \vec{k})$ coordinate frame around the $\vec{k}$ axis until the $\vec{j}$ axis is aligned with the normal vector at $S_{0}$. In this new orthonormal coordinate system $(\vec{i}, \vec{j}, \vec{k})$, the unit normals at $S_{0}$, $S_{r}$, and $S_{l}$ are $(0,1,0),(\sin \delta, \cos \delta, 0)$, and $(-\sin \delta, \cos \delta, 0)$, respectively, where $\delta$ is the angle made by the intersecting tangent planes at $S_{r}$ (or $S_{l}$ ) and $S_{0}$.

We are interested in the intensity values at $S_{r}, S_{l}$, and $S_{0}$, assuming diffuse reflectance and constant diffuse albedo. Let $\vec{L}=\left(e_{1}, e_{2}, e_{3}\right)$ be the unit point source directional vector. The image intensity values at $C_{0}, C_{r}$, and $C_{l}$ are directly proportional to the cosine of the angle between the normal vector at $S_{0}, S_{r}$, and $S_{l}$, respectively, and $\vec{L}$.

Without loss of generality, assume the intensity of the incident light and the diffuse reflectance cocfficient of the surface are equal to 1 . Thus, the intensity value at a point is equal to the cosine of its normal vector with the light source vector. The reflectance equations for $S_{0}, S_{r}$, and $S_{l}$ are then simply

$$
\begin{gathered}
R_{C_{0}}=\frac{e_{2}}{\sqrt{e_{1}^{2}+e_{2}^{2}+e_{3}^{2}}} \\
R_{C_{0}}=\frac{e_{1} \sin \delta+e_{2} \cos \delta}{\sqrt{e_{1}{ }^{2}+e_{2}^{2}+e_{3}^{2}}} \\
R_{C_{1}}=\frac{-e_{1} \sin \delta+e_{2} \cos \delta}{\sqrt{e_{1}^{2}+e_{2}^{2}+e_{3}^{2}}}
\end{gathered}
$$

It is easy to show that the same intensity values given for the image points by equations $(6.1),(6.2)$ and (6.3) can be obtained by moving the point light source so that it is coplanar with the points $S_{0} . S_{r}$, and $S_{t}$ and then rescaling the intensity of the incident light by a factor of $\frac{\sqrt{e_{1}{ }^{2}+e_{2}^{2}}}{\sqrt{e_{1}^{2}+e_{2}{ }^{2}+e_{3}{ }^{2}}}$. This new imaginary point source directional vector is given by $\vec{L}^{\prime}=\left(e_{1}, e_{2}, 0\right)$; sec [7].

As we have already mentioned, the points $S_{0}, S_{r}$, and $S_{l}$ all lie on a geodesic of the surface. Since the new point light source is coplanar with the geodesic curve (where meridian tangents are parallel to the axis), it is easy to see that the tangent planes at these three points do not tilt towards or away from the $\vec{L}^{\prime}$; in gradient space terminology, $q=0$.

Without loss of generality (see [7]), we again assume that the intensity of the incident light for this new (imaginary) point light source is equal to $I$ and that the point light source directional vector is a unit vector. Equations (6.1)-(6.3) can then be rewritten as

$$
\begin{gathered}
R_{C_{0}}=\cos \alpha_{0}=e_{2} \\
R_{C_{0}}=\cos \alpha_{1}=e_{1} \sin \delta+e_{2} \cos \delta \\
R_{C_{1}}=\cos \alpha_{2}=-e_{1} \sin \delta+e_{2} \cos \delta
\end{gathered}
$$

Using the trigonometric identity $\cos (x+y)=\cos x \cos y-\sin x \sin y$ and equations (6.5) and (6.6) we obtain

$$
R_{C_{.}}=\cos \alpha_{1}=e_{2} \cos \delta-e_{1} \sin \delta
$$

and

$$
R_{C_{1}}=\cos \alpha_{2}=e_{2} \cos \delta+e_{1} \sin \delta
$$

We can then add the intensity values at the image points $C_{r}$ and $C_{l}$ to obtain 


$$
R_{C_{1}}+R_{C_{0}}=2 e_{2} \cos \delta
$$

Dividing the right side of equation (6.9) by the right side of equation (6.4) yields simply $2 \cos \delta$, where $\delta$ is the angle between the tangent plane at $S_{0}$ and $S_{r}$ (or $S_{l}$ ). So the value of $\delta$ can be obtained directly from the intensity values at $C_{0}, C_{r}$, and $C_{l}$. But knowing the angle $\delta$ between the tangent planes, and the angle $\theta$ that the image tangent at $C_{r}$ (or $C_{l}$ ) makes with $\vec{w}$ (e.g., the image tangent at $C_{0}$ ) allows us to compute the object tilt $\beta$ since $\cos \beta=\frac{\tan \theta}{\tan \delta}$.

This method, then, does not need to know the light source position, diffuse reflectance cocfficient of the surface, or intensity of the incident light. Also, it works equally well for multiple light sources (see [7]). Details and examples of the method are given in [7].

\section{Recovery of the 3D Axis Position}

In this section, we present an intensity-based method for the recovery of the 3D axis position. This method presumes, as in the method of the previous section, that the SHGC has an extrema of the sweeping rule. We presume a parametcrization of the SHGC as in equation (2.1), and since we assume the image axis has already been recovered in section 5 (as a prerequisite to ruling the SHGC image), we are trying to solve for the translation parameter of the axis in the $\vec{i}$ direction, given by the $t_{z}$ parameter of Equation (2.1). We assume, since the image axis has been recovered, that $t_{y}$ is known. Recovering the $t_{z}$ parameter will allow us to completcly describe the shape of the SHGC image (modulo scale) and the recovery algorithm will be complete. We also presume that the object being recovered has a planar top. Again, all projections are presumed to be orthographic, with the object having constant albedo and lambertian reflectance. Without loss of generality (see [7]), we assume the lambertian albedo $\lambda=1$ for the entire SHGC surface.

Consider the imaging scenario illustrated in figure 6. The light source is presumed to be above the object (with respect to the object-centered coordinate system). The light source vector can be decomposed initially into orthogonal clements, one vector in the direction of (parallel to) the SHGC axis $\vec{k}$, while the other vector is perpendicular to the SHGC axis (parallel to the cross-section plane). We call the light intensity vector parallel to the SHGC axis $I_{A}$ and the light intensity vector parallel to the cross-section plane $I_{P}$ (see ligure 6 ).

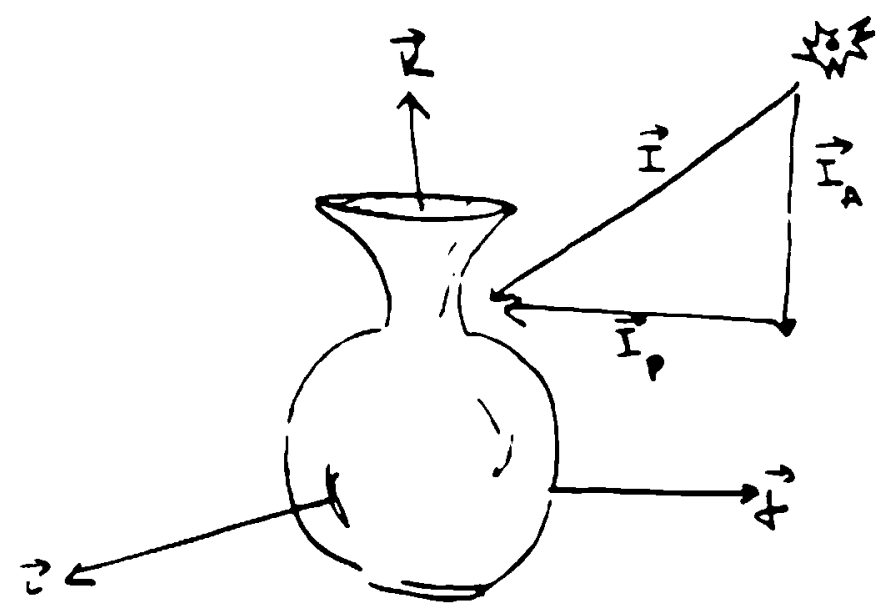

Figure 6. Imaging model for recovering the SHGC 3D axis position.

We now consider two points on the SHGC surface, $S_{G}$, and $S_{M_{0}}$, which correspond respectively to a point on a cross- sectional geodesic of the surface (where points on the geodesic can be found directly from image contour, as in the previous section), and a point on the same surface meridian as the selected 
geodesic point (see figure 7). We know that the tangent plane at a point on the surface geodesic is parallel to the SHGC axis [5], and we would like to determine, as a first step in recovering the 3D axis position, the angle that the tangent plane at $S_{M_{0}}$ makes with the tangent plane at $S_{G_{0}}$. To do this, we want to further divide the intensity ray into orthogonal components. Let $I_{M}$ be the component of $I_{P}$ (the vector parallel to the cross-section plane) in the direction of the surface meridian along which $S_{G_{\mathrm{a}}}$ and $S_{M}$, lie (see figure 7), and let $I_{T}$ be the the portion of $I_{P}$ orthogonal to $I_{M}$. It is intuitively obvious that $I_{T}$ will have no effect on the intensity values at either $S_{G_{0}}$ or $S_{M}$, since $I_{T}$ is parallel to the tangent planes at both points (see figure 7). In addition, assuming for now a single light source, the intensity at $S_{G}$, is clearly

$$
R_{G_{0}}=I_{M}
$$

while the intensity at $S_{M_{0}}$ is clearly given by

$$
R_{M_{0}}=\cos \phi I_{A}+\sin \phi I_{M}
$$

where $\phi$ is the angle the tangent plane at $S_{M_{0}}$ makes with the tangent plane at $S_{G_{n}}$, as shown in figure 8 .

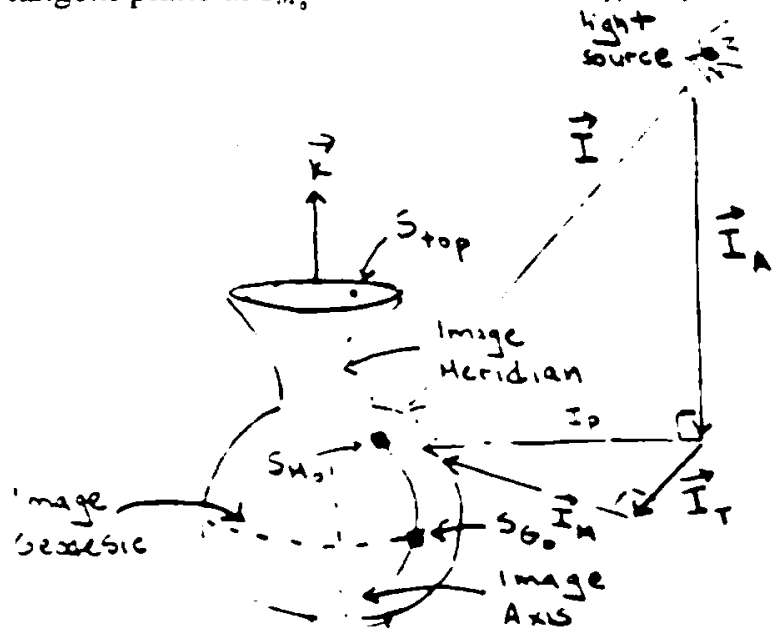

Figure 7. Decomposing the light intensity ray $\vec{i}$ into 2 components, parallel and orthogonal to the SHGC axis.

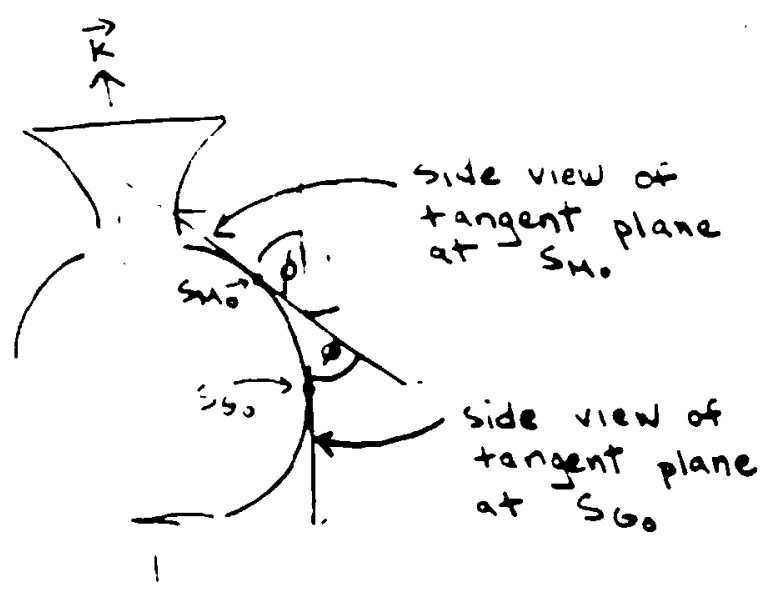

Figure 8. A side view of the SHGC meridian containing the points $S_{M_{0}}$ and $S_{G_{s}}$.

We now consider a third point on the SHGC surface, $S_{\text {top }}$, with intensity value given by

$$
R_{\text {lop }}=I_{A}
$$


As can be seen in figure 8 (sce also [7]), the intensity equation at $S_{\mathcal{M}_{0}}$ given by Equation (7.2) can be rewritten as

$$
R_{M_{0}}=\cos \phi R_{\text {top }}+\sin \phi R_{G_{0}}
$$

where $R_{\text {top }}, R_{G_{0}}$ and $R_{M_{0}}$ are simply the observed image intensities, respectively, at the points $S_{\text {Lop }}, S_{G_{0}}$, and $S_{M_{3}}$.

From equation (7.4) and the intensities at the points $S_{\text {top }}, S_{G_{\text {g }}}$, and $S_{M_{\mathrm{a}}}$, it is simple to obtain two closed form solutions for $\phi$, the angle between the tangent planes at $S_{G_{0}}$ and $S_{M_{2}}$, given by

$$
\phi_{1}=-\frac{R_{G_{0}} \sqrt{R_{G_{0}{ }^{2}+R_{\text {top }}{ }^{2}-R_{M_{0}}{ }^{2}}}+R_{\text {top }} R_{M_{0}}}{R_{\text {top }} \sqrt{R_{G_{0}{ }^{2}+R_{\text {top }}{ }^{2}-R_{M_{0}}{ }^{2}}-R_{G_{0} R_{M_{0}}}}}
$$

and

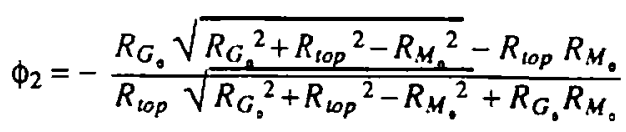

So we have two solutions for $\phi$, the angle between the tangent planes at $S_{G_{a}}$ and $S_{M_{0}}$. This turns out not to be a problem, as will soon be explained, and we are able to arrive at a unique solution for $\phi$.

Assuming without loss of generality (see section 2.2 ) that the only rotation of the object is towards (or away) from the viewer, we obtain the following equation for the normal of a point on the SHGC surface

$$
\left.\vec{N}=q^{\prime} \vec{i}-p^{\prime} \vec{j}++f^{\prime}\left(p^{\prime} q-\left(p+i_{x}\right) q^{\prime}\right)\right) \vec{k}
$$

From the equation for the normal, we can immediately obtain an equation for $\phi$, the angle the tangent plane at a point makes with the tangent plane of a point on a geodesic of the surface lying along the same meridian. This angle $\phi$ can be computed using the equation

$$
\tan \phi=\frac{\left(f^{\prime}\left(p^{\prime} q-\left(p+\ell_{x}\right) q^{\prime}\right)\right.}{v^{2}+p^{\prime 2}}
$$

But we can solve for $\phi$ directly from the intensity image using equations (7.5) and (7.6). In addition, we assume that the values of $p, q, p^{\prime}$, and $q^{\prime}$ are cither known or computable. This is a straightforward procedure as the tilt of the object has already been recovered (section 6), so that $p$ and $q$ at a point on the image are directly available from the image, while $p^{\prime}$ and $q^{\prime}$ can be computed using splines (see [7]). Thus, the only values in Equation (7.8) whose values are still not computable are $f^{\prime}$ and $t_{x}$.

We are interested in recovering $t_{x}$. To do so, we first rewrite Equation(7.8) with $f^{\prime}$ on the left side, such that

$$
f^{\prime}=\frac{\operatorname{lan} \phi \sqrt{q^{2}+p^{\prime 2}}}{\left(\left(p^{\prime} q\right)-\left(p+l_{x}\right) q^{\prime}\right)}
$$

To solve for $t_{x}$, we need to select another set of three points on the surface of the SHGC; we use the point $S_{\text {top }}$ again, and additionally sclect a geodesic point $S_{G_{1}}$ (on the same cross-section curve as $S_{G_{0}}$ ) and a point $S_{M_{1}}$ on the same meridian as $S_{G_{1}}$ and on the same cross-section curve as $S_{M_{0}}$. This is illustrated in figure 9. This set of three points, $S_{G_{1}}, S_{M_{1}}$, and $S_{\text {top }}$ will yield two solutions for $\phi$, as given by Equations (7.5) and (7.6). Selecting one of the solutions for $\phi$, the tangent plane angle, for the surface points at $S_{M}$, and $S_{M}$ and using Equation (7.9) gives us two equations for $f^{\prime}$, where the only unknown parameter on the right side of the equation is $t_{x}$. Since the points $S_{M_{1}}$ and $S_{M_{1}}$ lie on the same cross-section curve, the value 
of $f^{\prime}$ at both points is the same; thus, the right sides of the respective equations for $f^{\prime}$ generated using equation (7.9) must be equal. Setting them equal, we are able to get a solution for $t_{x}$ given by

$$
t_{x}=\frac{\tan \phi_{1} \sqrt{q_{1}^{\prime}{ }^{2}+p_{1}^{\prime}}\left(p_{0}^{\prime} q_{0}-p_{0} q_{0}^{\prime}\right)-\tan \phi_{0} \sqrt{q_{0}^{\prime}{ }^{2}+p_{0}^{\prime 2}}\left(p_{1}^{\prime} q_{1}-p_{1} q_{1}^{\prime}\right)}{\tan \phi_{0} p_{1}^{\prime} \sqrt{q_{0}{ }^{2}+p_{0}^{2}}-\tan \phi_{1} p_{0}^{\prime} \sqrt{q_{1}^{\prime 2}+p_{1}^{\prime 2}}}
$$

where $\phi_{0}$ is a solution for $\phi$ from the first set of meridian points $\left(S_{G_{0}}\right.$ and $\left.S_{M_{0}}\right), \phi_{1}$ is a solution for $\phi$ from the second set of meridian points $\left(S_{G_{1}}\right.$ and $\left.S_{M_{1}}\right)$, and $p_{i}, q_{i}, p_{i}{ }^{\prime}$, and $q_{i}{ }^{\prime}$ correspond to the cross-section functions (defined in equation (2.1)) and their derivatives at the point $S_{M_{M}}$. Since every term on the right side of equation (7.10) has already been computed, the value of $t_{x}$ can now also be computed.

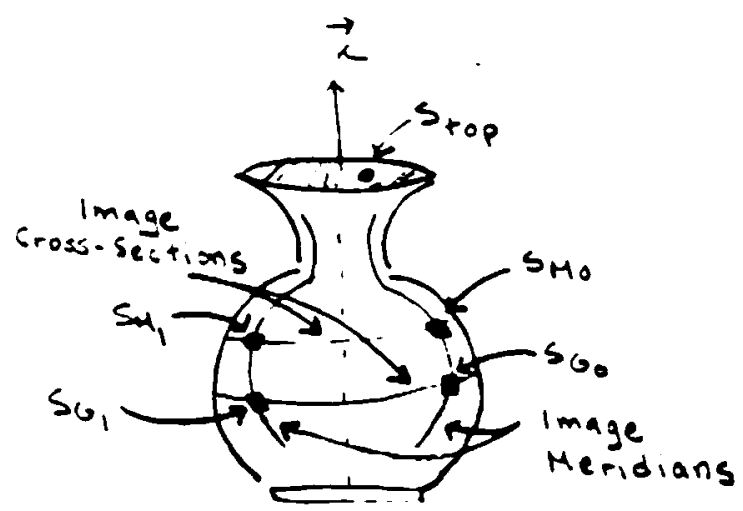

Figure 9. The SHGC surface points used to recover the 3D axis position.

To get a unique value for $t_{x}$, since there are two values of $\phi$ computed for each set of meridian points (corresponding to the two solutions for the tilt angle given in equations (7.5) and (7.6)), we take several sets of meridian points, where each set gives us four solutions for $t_{x}$, since there are four ways to select one $\phi$ value from each pair of $\phi$ valucs. Only one solution for $t_{x}$ will appear in all sets of solutions for $t_{x}$, and that is the desired value for $t_{x}$. It is easy 10 see [7] that this method works regardless of the number of light sources so long as a light source seen at point $S_{G}$ is also seen at point $S_{M_{1}}$. This does not seem unduly restrictive since $S_{M}$ can be chosen arbitrarily close to the surface geodesic point $S_{G_{i}}$.

Thus, we have demonstrated that all shape parameters of the SHGC (modulo scale) can be computed using constraints from both the contour and the intensity of the SHGC image. Examples of the method are given in [7].

\section{Conclusion and Future Work}

In this paper, an algorithm to uniquely recover the shape of an SHGC (modulo scale) was presented. It incorporates methods using both contour and intensity information. It is the first such algorithm known to the authors to recover SHGCs in a general, non-heuristic way. The methods that are intensity-based do not require a knowledge of the number of light sources, their positions, or intensities.

The problem of recovering the shape of an SHGC from contour images alone is inherenuly ill-posed. This point is formalized in the paper by defining classes of contour-equivalent SHGCs. We then demonstrated that the contour of an SHGC does not even determine the qualitative curvature of a surface region (such as whether it is clliptic or hyperbolic), let alone the quantitative Gaussian curvature. A method is then developed for ruling the image of an SHGC surface. We show that while a ruled SHGC image makes cerain parameters of the underlying SHGC explicit, such as the value of the sweeping rule at a 
point on the image, other parameters still need to be recovered, c.g., axis translation and object tilt.

This paper shows that additional constraints are required in order to recover SHGC shape from contour. One constraint available is that of intensity information. Next, we presented a method that makes use of both contour and intensity information to recover the tilt of an SHGC. The method detects image geodesics from extrema of distance contour points. Intensity values of certain points along the image geodesic are then used to compute the angle between the tangent planes at their corresponding surface points. The angle made by the tangent planes can then be used to recover the tilt of the SHGC vis a vis the viewer reference frame. Then, the final shape parameter (modulo scale) was recovered in section 7 , location of the 3D axis position, using the intensity values at several points $(2$ sets of meridian points and a point from the top cross-section plane). Thus, a complete algorithm for the recovery of the shape of an SHGC from its intensity image was presented.

Further research in this area will take several tracks. First, the algorithm presented in this paper, already tested experimentally, needs to be incorporated into a real-world system robust under noisy imaging conditions. A second direction for further research is to remove some of the restrictions on the current method, e.g., lamberian reflection and an extrema of the sweeping rule. Finally, the algorithm to recover SHGCs presented in this paper should be extended (if possible) to include a broader class of GCs, such as one that allows for different sweeping rules along onthogonal directions of the cross-section curve.

\section{Acknowledgements}

Support for this work was provided in part by the Advanced Research Projects Agency of the Department of Defense under contract \#NO0039-84-C-0165 and by the National Science Foundation Grant \#IRI8800370. Thanks to Cliff Beshers for his graphics package used in generating some of the images for this paper.

\section{References}

[1] Binford, T. O., Visual Perception by computer, Proceedings IEEE Conf. on Systems and Control, Miami, , December, 1971.

[2] Brady. J.M., and Yuille, A., An extremum principle for shape from contour, MIT, AI Lab., MIT-AIM 711, 1983.

[3] Brady, J.M., Ponce, J., Yuille, A., Asada, H., Describing Surfaces, in Proceedings of the 2nd Int' ' Symposium on Robotics Research, Harasuja and Inoue (ed.). MIT Press, 1985.

[4] Brooks, R. A., Symbolic reasoning among 3-D models and 2-D images, Artificial Intelligence 17, 285-348, 1981.

151 do Carmo, M.P., Differential geometry of curves and surfaces, Prentice-Hall, Inc., 1976.

[6] Fearing, F.S., Tactile sensing, perception, and shape interpretation, PhD thesis, Dept. of Electrical Engineering, Stanford University, Dec. 1987.

Gross. A.D., Recovery of straight homogeneous generalized cylinders from a single intensity view, CS Technical Repor CUCS-461-89, Columbia University, 1989.

Gross, A.D., Boult, T.E., Straight Homogeneous Generalized Cylinders: Analysis of Reflectance Propertics and a Necessary Condition for Class Membership, Darpa IU Workshop. Palo Alto, CA, 1989. 
[9] Horaud, R., and Brady, J.M., On the geometric interpretation of image contours, in Proc. First Int'l Conference on Computer Vision, London, U.K., June 1987.

[10] Koenderink, J.J., What does occluding contour tell us about solid shape?, Perception, vol. 13. pages. 321-330, 1984.

[11] Marr, D., Analysis of occluding contour, Proc. of Royal Society of London B-197, pp. 441475, 1977.

[12] Marr, D., Vision, SanFrancisco, CA, WH Frecman, 1982.

[13] Nalwa, Vic, Line-drawing interpretation: Bilateral symmetry, in Proceedings of the Image Understanding Workshop, vol. 2, Feb., 1987, pp. 956-967.

[14] Ponce, J., Chelberg, D., and Mann, W., Invariant propertics of the projections of straight homogencous Generalized Cylinders, in Proceedings of the First International Conference on Computer Vision, 1987.

[15] Ponce, J., Straight Homogeneous Generalized Cylinders: Differential Gcometry and Uniqueness Results, in Proceedings of Computer Vision and Pattern Recognition Conference, 327334, June 1988.

[16] Rao, K., and Medioni, G., Useful geometric properties of the generalized cone, in Proceedings of the Conference on Computer Vision and Pattern Recognition, June 5-9, 1988, pp. 276- 281.

[17] Richetin, M. Dhome, M., and LaPreste. J.T., Inverse perspective Iransform from zerocurvature curve points application to the localization of some generalized cylinders, in Proceedings of the 1989 Computer Vision and Pattern Recognition Conference, San Diego, Califomia, June 4-8, 1989.

181 Shafer. S., Shadows and silhouettes in computer vision, Kluwer Acad Publishers, Boston, 1985.

[19] Ulupinar, F., and Nevatia, R., Using Symmetries for Analysis of Shape from Contour, in Proceedings of the Second International Computer Vision Conference, 414-426, Dec. 5-8. 1988.

[20] Wciss, I., 3-d shape representation by contours, Computer Vision, Graphics, and Image Processing 41, 80-100, 1988. 